Erratum

\title{
Erratum to "Subamolide A Induces Mitotic Catastrophe Accompanied by Apoptosis in Human Lung Cancer Cells"
}

\author{
Jen-Yu Hung, ${ }^{1,2}$ Ching-Wen Wen, ${ }^{3}$ Ya-Ling Hsu, ${ }^{3}$ En-Shyh Lin, ${ }^{4}$ Ming-Shyan Huang, \\ Chung-Yi Chen, ${ }^{5}$ and Po-Lin Kuo ${ }^{6,7,8}$ \\ ${ }^{1}$ Division of Pulmonary and Critical Care Medicine, Kaohsiung Medical University Hospital, Kaohsiung 807, Taiwan \\ ${ }^{2}$ Department of Internal Medicine, Kaohsiung Municipal Ta-Tung Hospital, Kaohsiung 801, Taiwan \\ ${ }^{3}$ Graduate Institute of Medicine, Kaohsiung Medical University, Kaohsiung 807, Taiwan \\ ${ }^{4}$ Department of Beauty Science, National Taichung University of Science and Technology, Taichung 403, Taiwan \\ ${ }^{5}$ School of Medical and Health Sciences, Fooyin University, Kaohsiung 831, Taiwan \\ ${ }^{6}$ Institute of Clinical Medicine, College of Medicine, Kaohsiung Medical University, Kaohsiung 807, Taiwan \\ ${ }^{7}$ Cancer Center, Kaohsiung Medical University Hospital, Kaohsiung 807, Taiwan \\ ${ }^{8}$ Department of Medical Research, Kaohsiung Medical University Hospital, Kaohsiung 807, Taiwan
}

Correspondence should be addressed to Chung-Yi Chen; xx377@mail.fy.edu.tw and Po-Lin Kuo; kuopolin@seed.net.tw

Received 9 July 2013; Accepted 19 August 2013

Copyright $\odot 2013$ Jen-Yu Hung et al. This is an open access article distributed under the Creative Commons Attribution License, which permits unrestricted use, distribution, and reproduction in any medium, provided the original work is properly cited.

There are some errors that occurred during uploading Figures 5(a), 6(b), 6(e), and 7(b). The following are the corrected figures. 


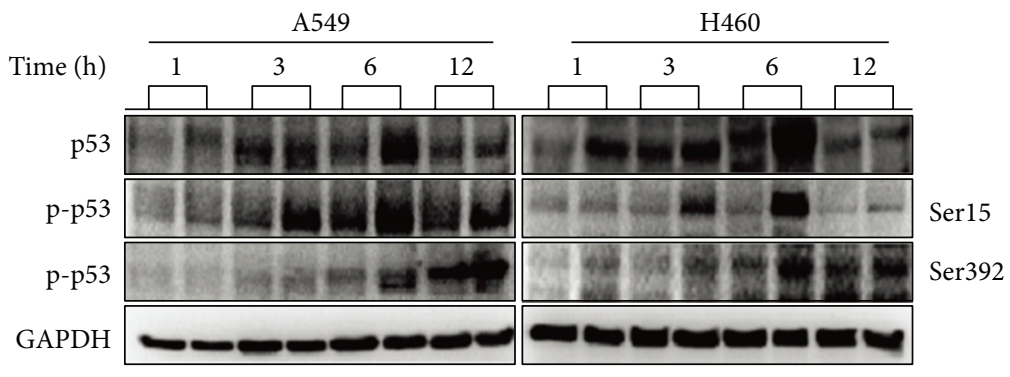

(a)

FIGURE 5

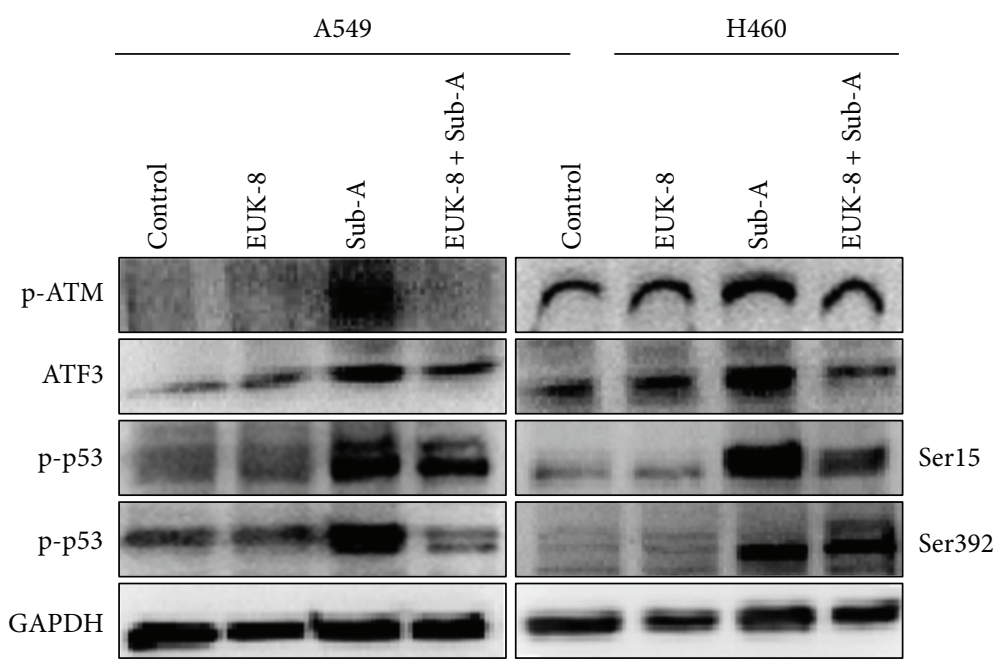

(b)

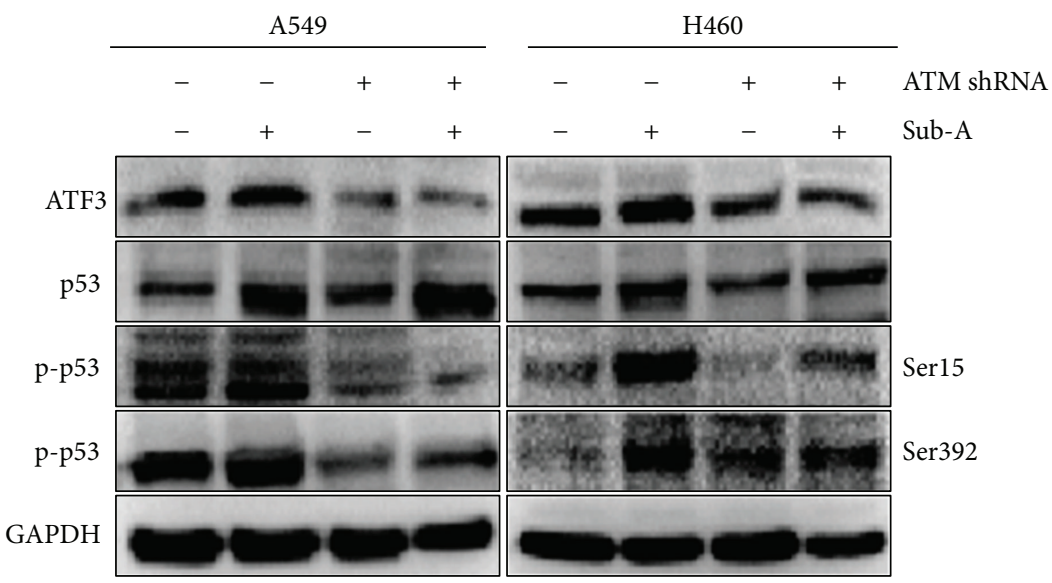

(e)

FIGURE 6 


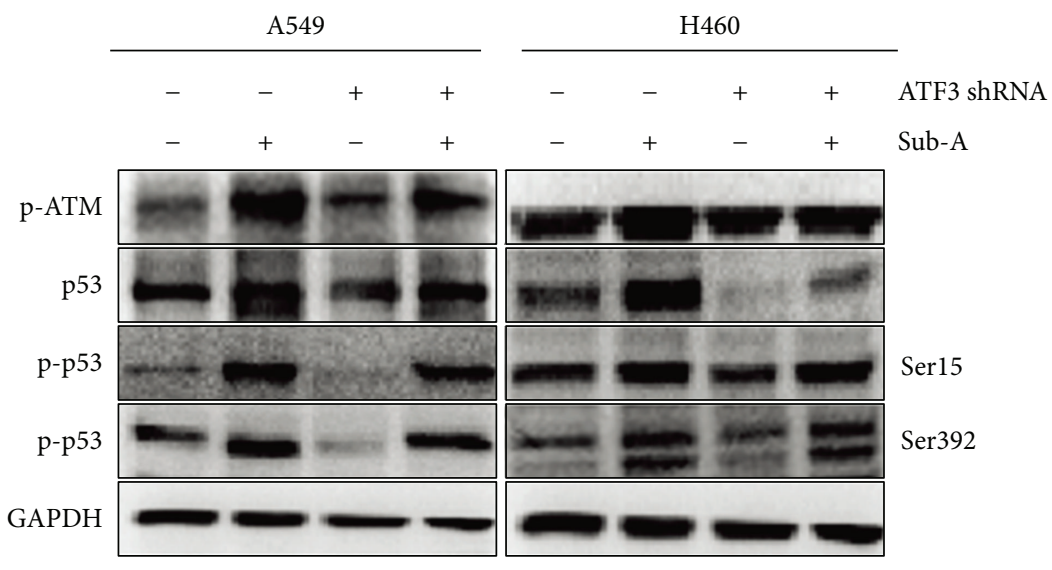

(b)

Figure 7 


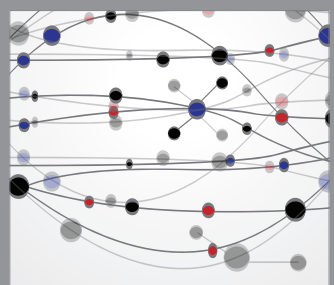

The Scientific World Journal
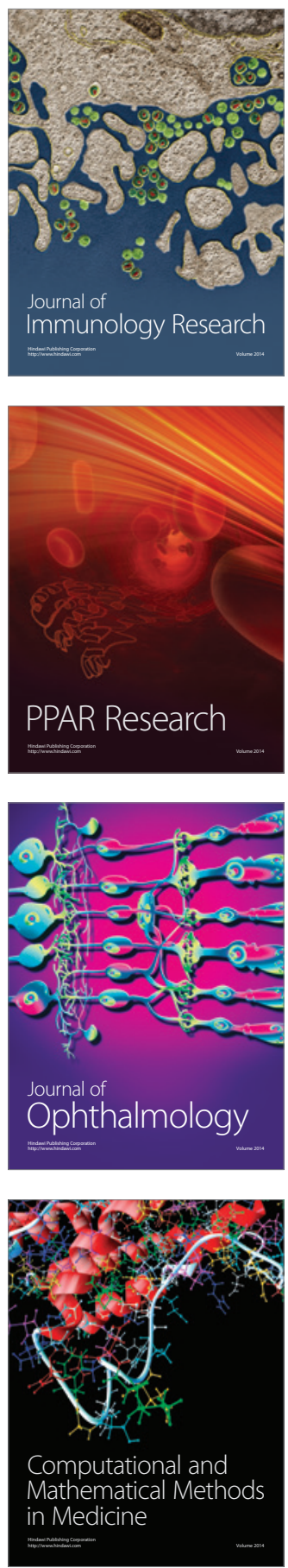

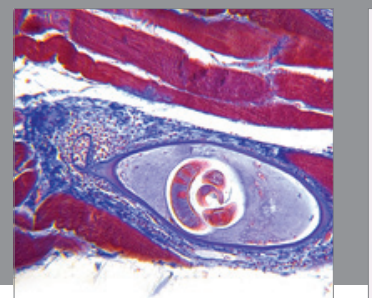

Gastroenterology

Research and Practice
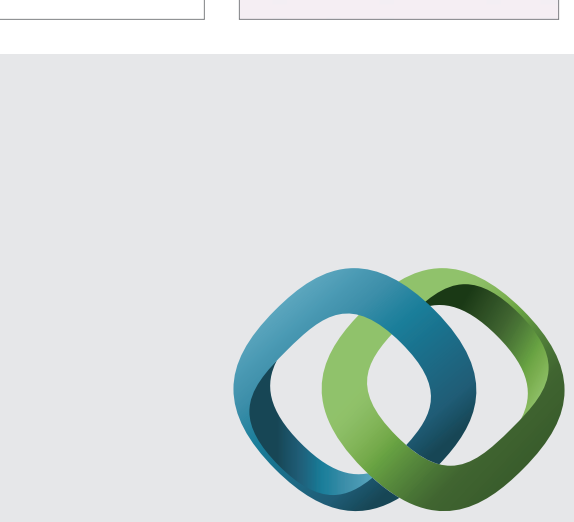

\section{Hindawi}

Submit your manuscripts at

http://www.hindawi.com
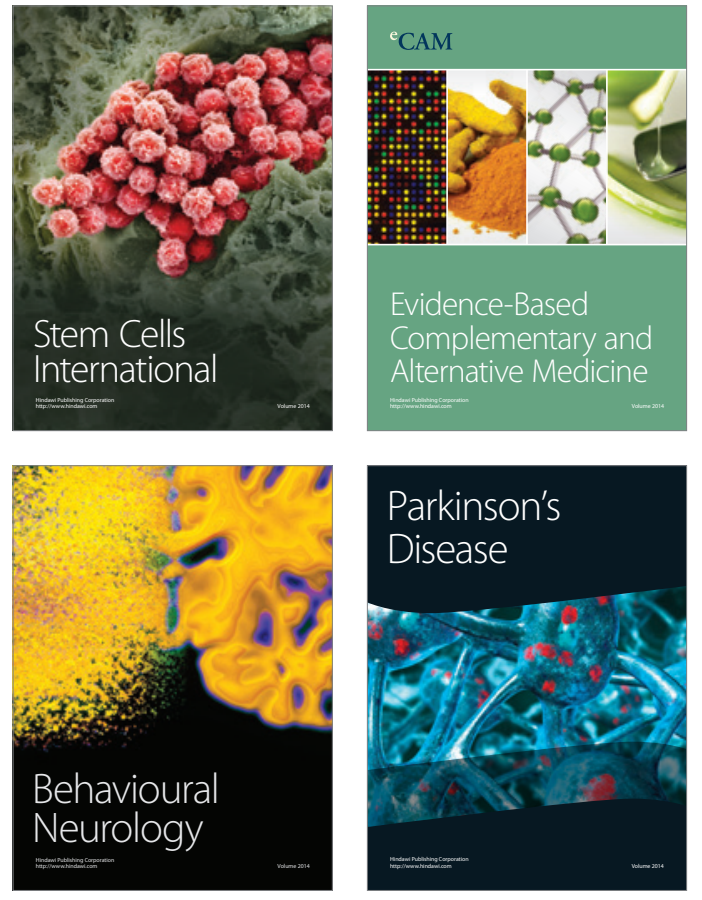
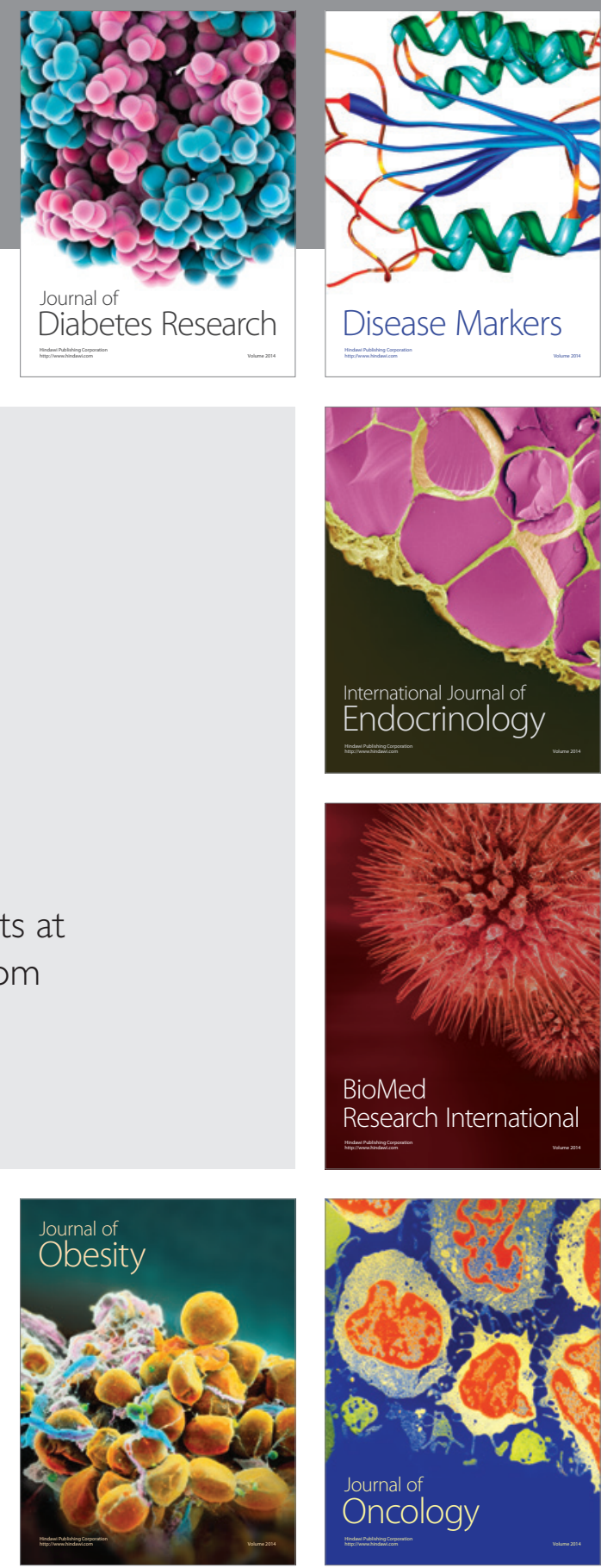

Disease Markers
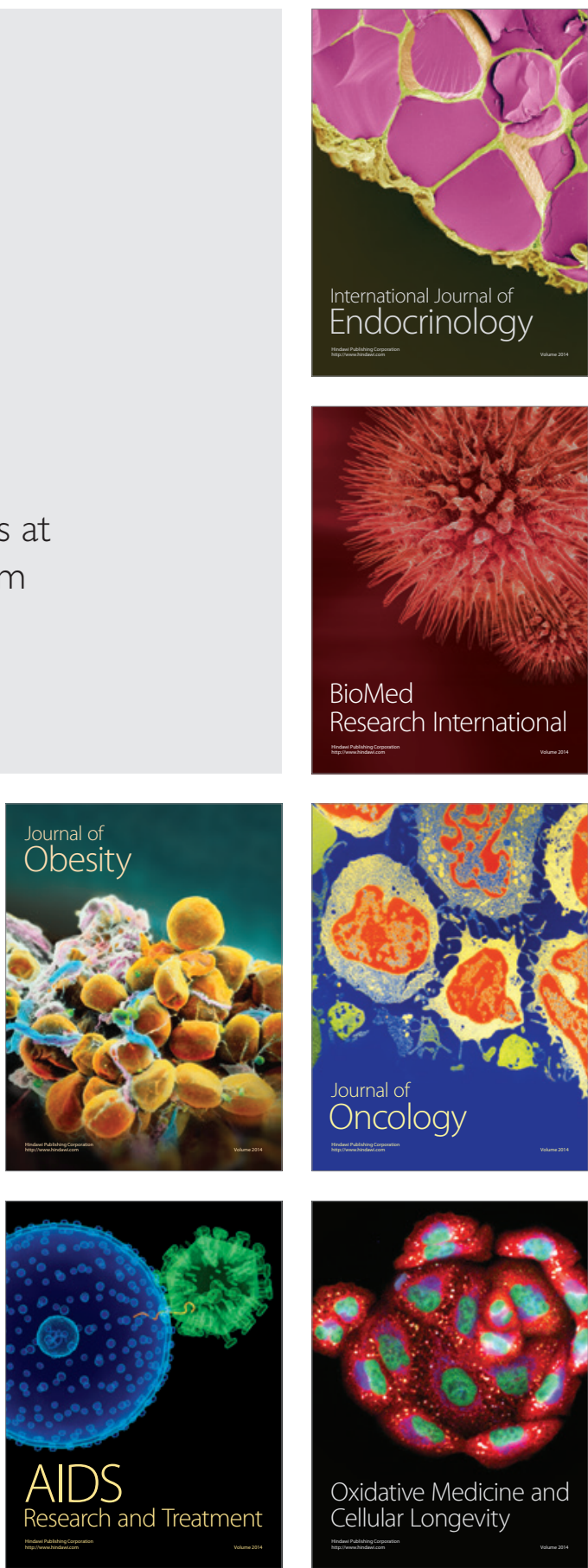